\title{
Model-independent and fast determination of optical functions in storage rings via multiturn and closed-orbit data
}

\author{
Bernard Riemann, ${ }^{*}$ Patrick Grete, and Thomas Weis \\ Zentrum für Synchrotronstrahlung (DELTA), Technische Universität Dortmund, \\ Maria-Goeppert-Mayer Straße 2, 44227 Dortmund, Germany
}

(Received 23 December 2010; published 28 June 2011)

\begin{abstract}
Multiturn (or turn-by-turn) data acquisition has proven to be a new source of direct measurements for Twiss parameters in storage rings. On the other hand, closed-orbit measurements are a long-known tool for analyzing closed-orbit perturbations with conventional beam position monitor (BPM) systems and are necessarily available at every storage ring. This paper aims at combining the advantages of multiturn measurements and closed-orbit data. We show that only two multiturn BPMs and four correctors in one localized drift space in the storage ring (diagnostic drift) are sufficient for model-independent and absolute measuring of $\beta$ and $\varphi$ functions at all BPMs, including the conventional ones, instead of requiring all BPMs being equipped with multiturn electronics.
\end{abstract}

DOI: 10.1103/PhysRevSTAB.14.062802

PACS numbers: 29.20.db, 29.27.Fh, 29.85.Fj

\section{INTRODUCTION}

Measuring optical functions in the storage rings is an essential part of beam diagnostics (see Ref. [1] for a recent review). However, measurements of the $\beta$ function have to cope with several kinds of intricacies. Among them are, for instance, the long duration of measurements with disruptive influence on the beam (e.g. if the $\beta$ function is measured via alteration of quadrupole strengths, see Ref. [2]), dependency on the quality of the lattice model of the storage ring, and high financial effort if many multiturn beam position monitors (BPMs) are used.

In this article, a cost-effective, model-independent, fast, and nondisruptive method for determining the $\beta$ function absolutely at all BPMs along the ring is presented. It employs a combination of only two multiturn-capable BPMs and two (only horizontal motion) respectively four (full transverse motion) correctors for small closed-orbit perturbations. In this paper, a proof of principle of this new method is given via measuring the horizontal $\beta$ and $\varphi$ function.

This paper is organized as follows. In Sec. II, the theoretical basis of the used method is presented, in Sec. III the experiments are described and discussed. Finally, in Sec. IV a conclusion and outlook is given. In addition, an appendix with the generalization of the presented method to full transverse motion and betatron coupling is provided.

\section{THEORY}

For uncoupled betatron oscillations, there are two independent trajectories possible in each plane. Because of

\footnotetext{
*bernard.riemann@tu-dortmund.de

Published by the American Physical Society under the terms of the Creative Commons Attribution 3.0 License. Further distribution of this work must maintain attribution to the author(s) and the published article's title, journal citation, and DOI.
}

elementary relations between betatron oscillation and closed-orbit perturbation, there are also two equivalent closed-orbit trajectories (see Ref. [3]), corresponding to dipole perturbations at two arbitrary positions in the ring.

These perturbed closed-orbit trajectories can be directly linked to the betatron oscillations if the optical functions at the two dipole corrector positions are known. If the correctors are inside a drift space with multiturn BPMs at its ends, this information can be obtained experimentally. Hence, the method proposed here consists of three consecutive steps: (1) determination of the optical functions within a drift space via multiturn BPMs (see Sec. II A); (2) application of small closed-orbit perturbations using two correctors inside the drift space; and (3) obtaining the response to this closed-orbit perturbations and determining the $\beta$ function and phase with it (see Sec. II B).

\section{A. Deriving the optical functions of a drift space with multiturn BPMs}

If there is just a drift space between two multiturn BPM positions (or anything else with a known transfer matrix-a drift space is the most simple case), it is possible to determine the absolute $\beta$ function at all multiturn BPM positions in the ring because one Poincare section in the transverse phase space becomes measurable (a similar argument is also used in Ref. [4]).

A short review of the technique described in Ref. [5] for the transverse phase space, including linear betatron coupling, is given. For simplicity, this technique is demonstrated in the $x-s$ or ring plane with uncoupled betatron motion. However, it is straightforward to include vertical motion $y$ and betatron coupling in this approach (see the Appendix).

The transverse position $x_{n j}$ of the undamped beam centroid at the longitudinal position of the BPM $j$ after a short transverse kick and $n$ turns around the ring can be written (see Ref. [6]) as 


$$
\begin{aligned}
& x_{n j}=\sqrt{\Upsilon_{x} \beta_{j}} \cos \left[\varphi_{j}+\mu_{x} n\right], \\
& x_{n j}^{\prime}=-\sqrt{\frac{\Upsilon_{x}}{\beta_{j}}}\left[\alpha_{j} \cos \left(\varphi_{j}+\mu_{x} n\right)+\sin \left(\varphi_{j}+\mu_{x} n\right)\right]
\end{aligned}
$$

with the horizontal Courant-Snyder invariant $\Upsilon_{x}$, the Twiss parameters $\alpha_{j}, \beta_{j}$, the betatron phase $\varphi_{j}$, and the horizontal betatron phase advance per turn $\mu_{x}=2 \pi Q_{x}$. Since these real sequences $x, x^{\prime}$ have a sinusoidal form, each sequence can be described using only one phasor or complex Fourier component via

$$
\begin{aligned}
X_{j} & =\sqrt{\Upsilon_{x} \beta_{j}} e^{\mathrm{i} \varphi_{j}}, \\
X_{j}^{\prime} & =-\sqrt{\frac{\Upsilon_{x}}{\beta_{j}}}\left[\alpha_{j}-\mathrm{i}\right] e^{\mathrm{i} \varphi_{j} .}
\end{aligned}
$$

Phasors can in principle be calculated by using discrete Fourier transform on a sequence and dividing the component corresponding to $\mu_{x}$ by the sequences length. Since a measurable sequence always has a finite length, the occurring leakage effect must be taken into account which is done at the end of this section.

In a drift space of length $l$ with BPMs $j=1,2$ at its ends, there is an elementary relation between the phase space variables, i.e.,

$$
x_{n 1}^{\prime}=x_{n 2}^{\prime}=\frac{x_{n 2}-x_{n 1}}{l}
$$

which is also applicable to their phasors. Thus, one can determine $X_{1 / 2}^{\prime}$ from experimental data in a drift space. Consider the complex numbers $X_{1}$ and $X_{1}^{\prime}$ as vectors $\vec{X}_{1}$ and $\vec{X}_{1}^{\prime}$ in the complex plane and $A$ as the area spanned up by them (see Fig. 1). It is crucial for understanding the special role of drift spaces in this approach to recognize that $A$ equals the Courant-Snyder invariant (see also Ref. [5]), i.e.,

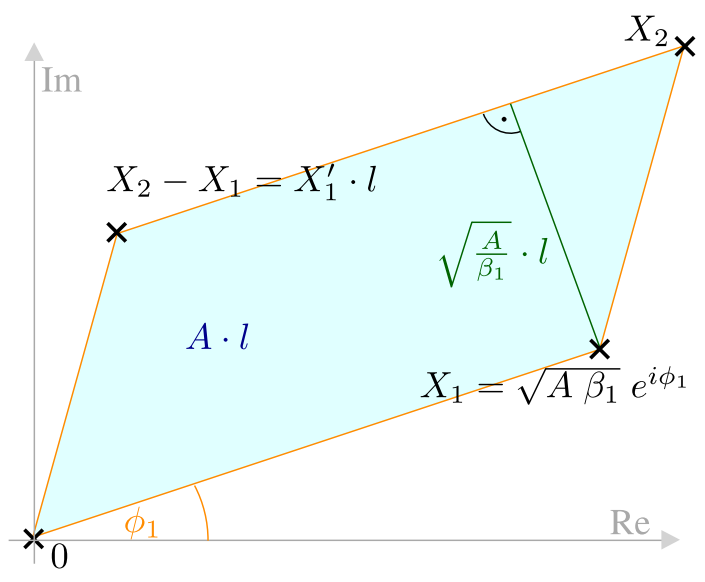

FIG. 1. Sketch of geometrical relations between phasors. $A$ can be determined using elementary calculations.

$$
\begin{aligned}
A & =\left|\vec{X}_{1} \times \vec{X}_{1}^{\prime}\right|=\operatorname{Im}\left(X_{1}^{*} \cdot X_{1}^{\prime}\right)=\frac{1}{l}\left|\vec{X}_{1} \times\left(\vec{X}_{2}-\vec{X}_{1}\right)\right| \\
& =\frac{1}{l} \operatorname{Im}\left(X_{1}^{*} \cdot X_{2}\right)=\sqrt{\Upsilon_{x} \beta_{1}} \sqrt{\frac{\Upsilon_{x}}{\beta_{1}}}=\Upsilon_{x} .
\end{aligned}
$$

Hence, the Courant-Snyder invariant $A$ can be determined using Eq. (4) with multiturn data. This enables the absolute determination of the horizontal $\beta$ function at all multiturn BPMs $j$ via Eqs. (2). The $\beta$ function has a parabolic form inside the drift space, and the optical parameter $\alpha_{1}=-\frac{1}{2} \beta_{1}^{\prime}$ is also determined for the drift space case via

$\vec{X}_{1} \cdot \vec{X}_{1}^{\prime}=\operatorname{Re}\left(X_{1}^{*} \cdot X_{1}^{\prime}\right)=-\sqrt{\Upsilon_{x} \beta_{1}} \sqrt{\frac{\Upsilon_{x}}{\beta_{1}}} \operatorname{Re}\left(\alpha_{1}-\mathrm{i}\right)=-\Upsilon_{x} \alpha_{1}$.

Thus, the $\beta$ and $\varphi$ functions can be determined continuously inside the whole drift space using the $\beta_{1 / 2}, \alpha_{1}$ values of the BPM positions at its ends.

One merit of analyzing multiturn data and determining the Courant-Snyder invariant $Y$ (this applies to both $Y_{x}$ and $Y_{y}$ ) in the frequency domain is the insensitivity of this method against decoherence damping phenomena of the beam centroid motion. This requires that the value of $Y$ can still be approximated as constant within one turn $n$. Although the oscillation envelope $\sqrt{\Upsilon(n) \beta_{j}}$ is time dependent in this case [see Eq. (1)], the dependence of $x_{n j}$ on $Y(n)$ is the same for any given turn $n$ at all BPM positions $s_{j}$ in the ring (a similar argument is also used in Ref. [7]). According to the convolution theorem, the betatron lines in the different BPM spectra $j$ are then convolved and thus broadened with the same Fourier spectrum of $\sqrt{\Upsilon(n)}$, and the maximum values of all lines are reduced (and rotated) by the same complex factor.

Thus, if the Fourier components with maximal amplitude are taken as phasors for the described calculations, the damping only affects the measured $A$, but not the measured local $\beta_{j}, \varphi_{j}$ values. The same argumentation holds for the leakage effect, since its definition is based on convolution of an infinite sequence with a window function. In addition, one can use ordinary Fourier components of the Fast Fourier Transform algorithm as phasors, since their global scaling (e.g. division by sequences length) also only affects $A$.

\section{B. Closed-orbit perturbation with known values for two correctors}

The well-known description of the linear orbit response in one plane reads (see, e.g., in Ref. [8])

$$
\vec{x}=\boldsymbol{R} \vec{\theta}
$$

with

$$
R_{j k}=C_{Q \sqrt{\beta_{j} b_{k}}} \cos \left(\varphi_{j}-\tilde{\psi}_{k}\right)
$$


where $\vec{\theta}$ is a vector which contains all applied dipole kick angles, $\vec{x}$ contains the deviation at all BPMs $j$ from the ideal orbit, and $\boldsymbol{R}$ is called orbit response matrix (ORM). $C_{Q}^{-1}=2 \sin \left(\mu_{x} / 2\right)$ is assumed as a ring-global nonzero factor. To avoid index confusion, the beta and phase values at the corrector with index $k$ are denoted with $b_{k}, \psi_{k}$ while the corresponding functions at the BPM with index $j$ are denoted with $\beta_{j}, \varphi_{j}$ as usual. In addition,

$$
\tilde{\psi}_{k}:= \begin{cases}\psi_{k}+\mu_{x} / 2 & \text { for } \varphi_{j}>\psi_{k}, \\ \psi_{k}-\mu_{x} / 2 & \text { for } \varphi_{j}<\psi_{k}\end{cases}
$$

is defined for simplicity.

By inspection of Eqs. (7) and (1a), one sees that the expression for the transverse trajectory of a closed orbit under the influence of a small dipole perturbation is similar to that of a betatron oscillation (see also Ref. [2]). One difference is a phase discontinuity $\mu_{x}$ at the position of the dipole [see Eq. (8)] which makes the oscillation periodic in the accelerator path length. In addition, $C_{Q}$ incorporates the sensitivity of the orbit on perturbations and is related to tune resonances.

For a closed-orbit-only determination of the lattice parameters, the values of $b, \psi$ at the corrector positions are only known approximately by a model lattice, and thus one will try to match the model $\boldsymbol{R}$ matrices to the measured $\boldsymbol{R}$ matrices which is usually done by orbit response matrix analysis and algorithms like LOCO [9].

If the optical functions $b_{k}, \psi_{k}$ at the position of two dipole correctors are known, the optical functions at all BPM positions can be directly determined by using only the two columns of the response matrix given by $R_{j 1}$ and $R_{j 2}$. This is indeed the case if the correctors are placed in a drift space with multiturn BPMs at its edges (see Sec. II A). Their Twiss parameters can be used in a straightforward manner to calculate the optical functions in the drift space and thus also at the corrector positions.

Hence, a system of two equations (7) with $k=1,2$ is obtained with two unknowns for each BPM $j$. This system is solvable with practically avoidable exceptions that are addressed below. By using the relation

$$
\sqrt{\frac{b_{2}}{b_{1}}} \frac{R_{j 1}}{R_{j 2}}=\frac{\cos \left(\varphi_{j}-\tilde{\psi}_{1}\right)}{\cos \left(\varphi_{j}-\tilde{\psi}_{2}\right)}=\frac{\cos \left(u_{j}+\Delta \psi\right)}{\cos u_{j}}
$$

with the unknown phase $u_{j}:=\varphi_{j}-\tilde{\psi}_{2}$ and the phase difference $\Delta \psi:=\tilde{\psi}_{2}-\tilde{\psi}_{1}=\psi_{2}-\psi_{1}$ of the two correctors, one gets

$$
\tan u_{j}=\frac{1}{\tan \Delta \psi}-\frac{1}{\sin \Delta \psi} \sqrt{\frac{b_{2}}{b_{1}}} \frac{R_{j 1}}{R_{j 2}} .
$$

Since the tangent function is not bijective over $2 \pi$, there is an ambiguity relating to the sign of the $R$ components if Eq. (10) is solved for $u_{j}$. In order to restore the uniqueness of the solution, the sign of $R_{j 2} \propto \cos u_{j}$ has to be included, and the expression for $u_{j}$ reads

$$
u_{j}=\arctan \left(\frac{1}{\tan \Delta \psi}-\frac{1}{\sin \Delta \psi} \sqrt{\frac{b_{2}}{b_{1}}} \frac{R_{j 1}}{R_{j 2}}\right)+ \begin{cases}\pi & \text { for } R_{j 2}<0 \\ 0 & \text { for } R_{j 2}>0 .\end{cases}
$$

Furthermore, one gets

$$
\beta_{j}=\frac{R_{j 2}^{2}}{b_{2} C_{Q}^{2}}\left(1+\tan ^{2} u_{j}\right)
$$

for the $\beta$ value at BPM $j$. In addition, it follows

$$
\varphi_{j}=u_{j}+\tilde{\psi}_{2}= \begin{cases}u_{j}+\psi_{2}+\mu_{x} / 2 & \text { for } \varphi_{j}>\psi_{2}, \\ u_{j}+\psi_{2}-\mu_{x} / 2 & \text { for } \varphi_{j}<\psi_{2}\end{cases}
$$

Equations (11)-(13) are sufficient to determine the respective optical functions at the positions of all BPMs.

From Eqs. (11) and (12), one can directly identify the main source of errors of the presented method, i.e., the response matrix elements $R_{j 1}, R_{j 2}$ have to be obtained accurately. This is hampered by any imperfection of determining the beam position (e.g. unaccounted pincushion distortion, pickup misalignment). Another source of errors is timing errors of the multiturn BPMs at the edges of the drift space (described in the measurement results) and the amount of data from the betatron oscillation retrievable by them (by influencing signal-to-noise ratio of the multiturn measurement).

Equation (11) takes a simpler form if $\Delta \psi=\pi / 2+n \pi$ with $n \in \mathbb{Z}$ is fulfilled. In this special case, the closed-orbit distortions are orthogonal which is the optimal case for the corrector positions.

A restriction is posed on the presented approach for $\Delta \psi=n \pi$. In this case, the method will not work, since the produced orbit perturbations are linearly dependent and no additional information can be retrieved using the second corrector. However, in most practical cases this can be circumvented by placing the dipole correctors in proper positions.

If a direct comparison with simulated multiturn data is desired, the data points corresponding to this measurement may be created by using Eq. (1a). It is also possible to construct these data points with less intermediate steps by calculating them using a tracked $\boldsymbol{F}_{j}$ matrix (see the Appendix).

It should be noted that, if there is no single drift space available where the two (respectively four) correctors can be placed, one can use up to two (respectively four) drift spaces (with multiturn BPMs at their edges) in which the correctors can be placed in arbitrary distribution. The necessary condition for the presented method is that the optical parameters $b_{k}, \psi_{k}$ at the positions of two horizontal (and, for full transverse motion, two vertical) dipole correctors can be determined with multiturn BPM data. Obviously, this increases the number of multiturn BPMs needed and, hence, also the monetary effort. 


\section{Normalizing the perturbation results using the multiturn drift measurement}

Up to this point, it was assumed that the dipole kick angle $\theta_{k}$ and therefore the kick-to-current ratio $C_{k}:=$ $\theta_{k} / I_{k}$ of the used corrector electromagnets is known. If this is not the case, Eq. (6) can be modified to

$x_{j}=\sum_{k} R_{j k} C_{k} I_{k}=\sum_{k} C_{Q} C_{k} \sqrt{\beta_{j} b_{k}} \cos \left(\varphi_{j}-\tilde{\psi}_{k}\right) I_{k}$.

The values of $\beta_{j}, \varphi_{j}$ are known for the two BPMs at the ends of one drift space by the multiturn measurement. Thus, one can determine the calibration factor $C_{Q} C_{k} \sqrt{b_{k}}$ by comparison of closed-orbit and multiturn measurement at the drift BPM positions. The absolute $\beta_{j}$ values can then be determined for all other BPMs using Eq. (12), even if the corrector kick-to-current ratios $C_{k}$ are not known beforehand. Via this method it is also possible to determine the value $C_{k}$ of the corrector magnet. In addition, also the two columns of $\boldsymbol{R}$ that correspond to the drift space correctors can be derived without knowing this scaling factor.

\section{MEASUREMENTS IN THE DELTA STORAGE RING}

\section{A. Experimental setup}

The synchrotron radiation facility DELTA (see Fig. 2), operated by the Center for Synchrotron Radiation, located at TU Dortmund University, consists of a linac, a fullenergy synchrotron, and a $1.5 \mathrm{GeV}$ electron storage ring with a circumference of $115.2 \mathrm{~m}$. The storage ring exhibits three insertion devices, i.e., the electromagnetic undulator $\mathrm{U} 250$, the permanent magnet undulator U55, and the superconducting asymmetric wiggler (SAW). There are 54

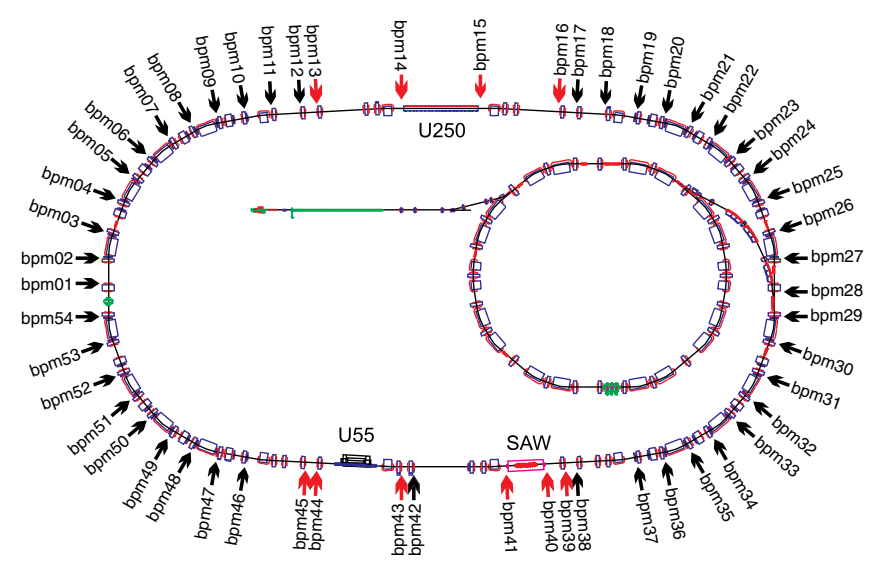

FIG. 2. Map of the DELTA synchrotron radiation facility, consisting of a linac, a full-energy synchrotron, and a storage ring with 54 BPMs installed (black and red arrows). Ten of them (red arrows) are capable of taking multiturn data at a sampling rate equal to the revolution frequency. For the measurements presented here, the U250 (drift) section between BPM 14 and BPM 15 is used. double-view BPMs installed in this ring, 44 work with analog conventional BPM electronics with a sampling rate of $10 \mathrm{~Hz}$ (black arrows in Fig. 2, [10]), while the remaining 10 BPMs are equipped with muliturn-capable readout electronics, using a sampling rate of $2.6 \mathrm{MHz}$ (red arrows in Fig. 2, [11]). Note that up to now, the multiturn capability of BPM 39-41 is not yet set up.

For the measurements presented here, the drift space with a length of 5.137 m between BPM 14 and BPM 15 is used. The electromagnetic undulator between these BPMs consists of 38 dipole coils, has a length of $4.875 \mathrm{~m}$, and was turned off during the measurements. Apart from the main coils of the undulator, there are also 38 smaller coils for beam correction purposes in horizontal direction. However, those correction coils remained unused until now, so they could be used for creating the necessary closed-orbit perturbations. This setup comprises a flexible length of the corrector distance so that a phase shift $\Delta \psi=$ $n \pi$ could be avoided. Via a MATLAB [12] script and an EPICS-based control system [13], a sinusoidal current with a frequency of approximately $0.1 \mathrm{~Hz}$ was applied subsequently on the first and the last correction coil of the undulator. For the multiturn measurements, a diagonal "slotted-pipe"-type kicker in the vicinity of BPM 3 was used [8]. The multiturn-capable BPMs were triggered upon kicker activation and recorded the transverse beam position for the next 2048 turns of the excited beam.

\section{B. Results of the measurements}

Together with the time for acquiring the turn-by-turn data, the whole measurement of the horizontal $\beta$ function takes approximately 40 seconds. With the method described above, the horizontal $\beta$ function and the phase values for several beam currents $I_{\text {beam }}(5-90 \mathrm{~mA})$ are calculated and several strengths of the closed-orbit perturbation for each beam current are used. The strength of the perturbation is controlled via the amplitude of the sinusoidal current $I_{\text {corr }}$ applied to the corrector coils which is set to $0.5 \mathrm{~A}, 1 \mathrm{~A}, 2 \mathrm{~A}$, and $3 \mathrm{~A}$. One is free to choose $\varphi=0$ at one arbitrary position in the ring. The phase $\varphi$ for all results presented here is gauged such that $\varphi=0$ is fulfilled at the beginning of the drift section.

An overview of the results for several beam currents with the storage rings standard lattice configuration is gained via Figs. 3 and 4. There, $\beta_{x}$ and $\varphi_{x}$ from the presented combined method (circles) in comparison with the given standard lattice model (continuous lines) and with the other multiturn BPMs (black crosses) are shown for $I_{\text {corr }}=3 \mathrm{~A}$ (Fig. 3) and $I_{\text {corr }}=0.5 \mathrm{~A}$ (Fig. 4), respectively. The presented combined method seems to work for the presented range of beam and corrector coil currents.

Measurement errors at high beam currents $I_{\text {beam }}>$ $100 \mathrm{~mA}$ are caused by signal overdrive of the BPM electronics (not shown). At DELTA, the amplification factors for the BPM pickup signals cannot be varied using the 


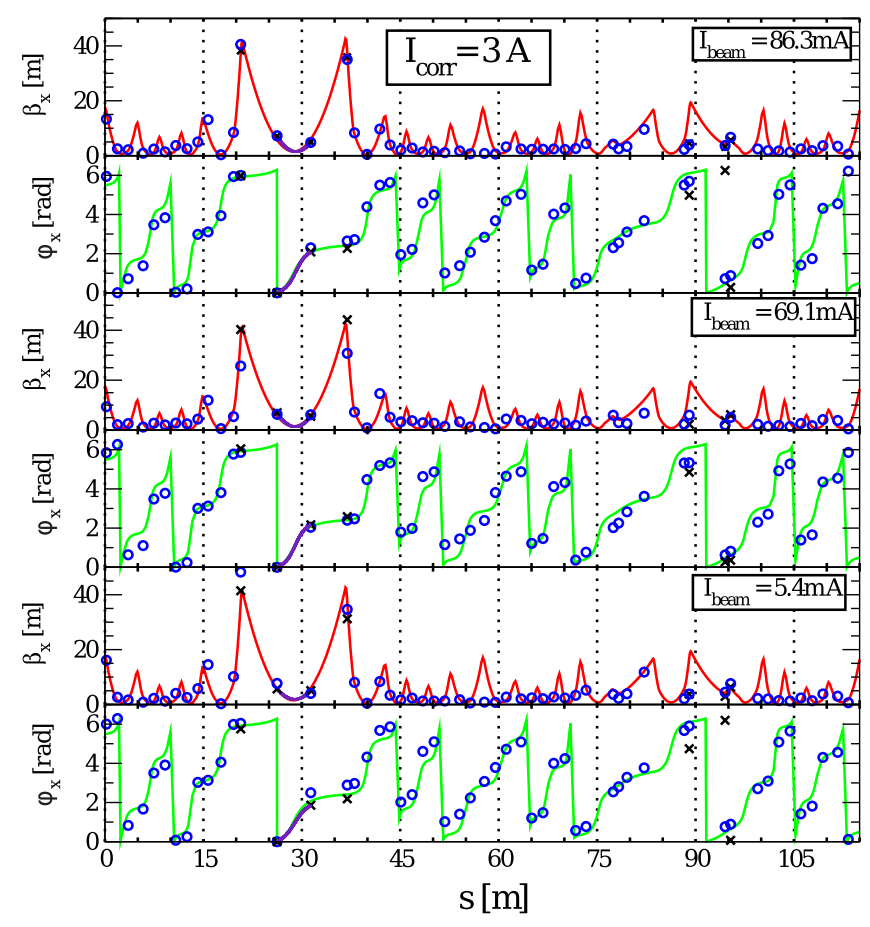

FIG. 3. Measurements of the horizontal $\beta$ function and phase for standard lattice configuration, $I_{\text {corr }}=3 \mathrm{~A}$ current at the corrector coils and several beam currents $I_{\text {beam }}$ (see legend). Shown are the theoretical $\beta$ function (red) and phase (green) taken from the given lattice model, the corresponding values from the seven multiturn BPMs (black crosses) and the results from the combined method (blue circles). In the drift space (around $s=30 \mathrm{~m}), \beta_{x}$ and $\varphi_{x}$ can be obtained continuously (indigo line).

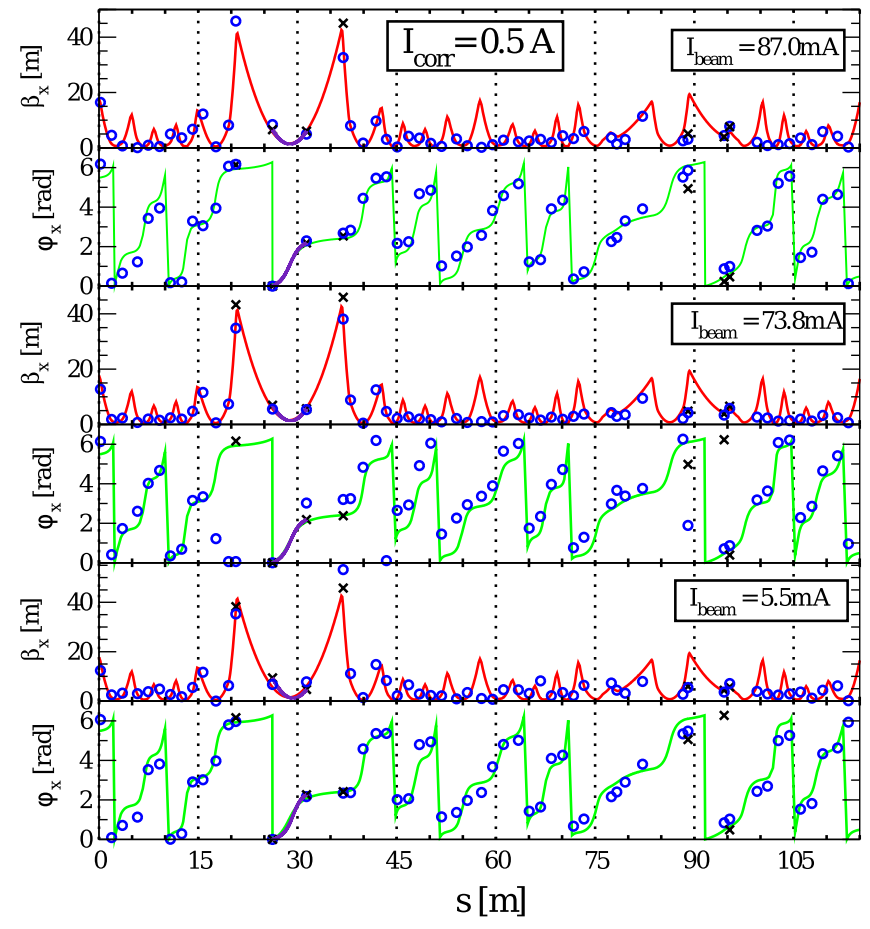

FIG. 4. Same measurement as in Fig. 3 with $I_{\text {corr }}=0.5 \mathrm{~A}$. control system and are thus constant during operation. This leads to systematic measurement errors at high beam currents. At low beam currents $I_{\text {beam }} \leq 5 \mathrm{~mA}$, the low signal-to-noise ratio may hamper the measurement of oscillation amplitudes and phases. This may also be the case for small oscillation amplitudes resulting from low $\beta$ functions at some BPMs.

For a detailed analysis, the case with $I_{\text {beam }}=78.8 \mathrm{~mA}$ beam current and $2 \mathrm{~A}$ current at the corrector coils shown in Fig. 5 is considered. Comparing those results with the results of the multiturn BPMs, one obtains deviations of at most $20 \%$ in $\beta_{x}$. The phase measurements fit the model better than the $\beta$ measurements. Comparing the presented combined method results with the lattice model curves, one obtains less relative deviation of at most $15 \%$.

The measured phase difference of the BPMs in the vicinity of $s \approx 95 \mathrm{~m}$ does not coincide with the lattice model. This phase difference is most likely caused by a timing (trigger) error which means that the position sequences of both BPMs are shifted about one turn against each other. At the horizontal fractional betatron tune $Q_{x}=0.16$, this leads to a phase measurement error of $\Delta \varphi \approx 1 \mathrm{rad}$ which fits to the observed deviation of the model curve (green) and multiturn result around this position.

Since these timing errors do not occur at a drift section BPM used for the combined method in this case, they do not modify the combined method results. This shows that only the two drift section BPMs have to be synchronized for correct combined method results, while an equivalent measurement using only multiturn data would show additional errors. Synchronization of many multiturn BPMs to the arrival of a particle bunch ensemble along a large path of a storage ring is a nontrivial task, while it is manageable to synchronize two BPMs to each other which are in vicinity.

The largest deviation occurs at $s=58 \mathrm{~m}$ where the beam is injected from the synchrotron. At DELTA, the

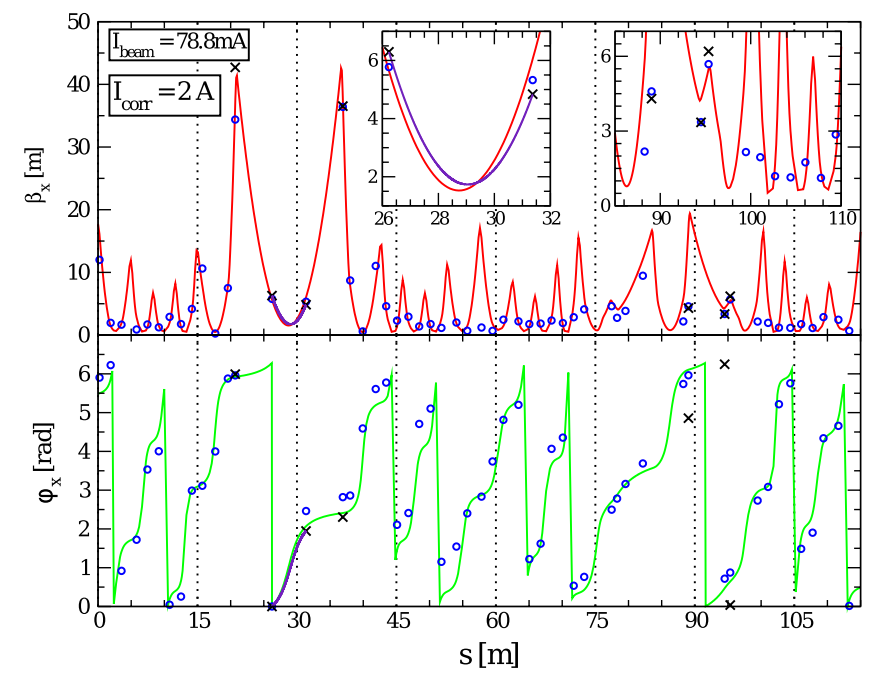

FIG. 5. Same measurement as in Figs. 3 and 4 for $I_{\text {beam }}=$ $78.8 \mathrm{~mA}$ and $I_{\text {corr }}=2 \mathrm{~A}$. 
strength of the installed kicker devices is not sufficient for injection, so an additional dc injection bump has to be created. Because of the large orbit deviation $(\approx 10 \mathrm{~mm})$ at the injection point from the middle of the beam chamber and due to the large pincushion distortion caused by this deviation, the closed-orbit perturbation is not measurable by the BPM at $s=58 \mathrm{~m}$. If the dc injection bump is removed, the measured value coincides with the theoretical curve (not shown). For the phase, one sees that the presented combined method does not have the problem of occurring phase jumps unless there is a timing difference between both drift section BPMs.

All mentioned errors in the presented results can be traced back to the general source of errors of the presented method (see Sec. II B), i.e., timing errors of the multiturn BPMs and an imperfect determination of the beam position at all BPMs.

\section{Comparison to quasistatic and multiturn-based methods}

The measurement approach presented in this paper is a combination of closed-orbit and multiturn-based techniques. Because of this combination, the following combination of advantages and disadvantages from both fields is achieved. The presented combined method provides a fast-but slower than pure multiturn measurementsdetermination of the $\beta$ function and phase advances. Since only two multiturn BPMs are used, only those two have to be synchronized by one trigger. This is obviously more stable than synchronizing all 54 BPMs in the whole ring-a source of instability which can be seen in the measurements of the phase advances by the multiturn BPMs as phase jumps (see around $s=90 \mathrm{~m}$ in Figs. 3 and 5). Those phase jumps are absent for the combined method introduced here, unless the two used multiturn drift BPMs are not properly synchronized to each other. In addition, the combined method provides an absolute determination of $\beta$ and $\varphi$. Since a drift space with known transfer matrix is used to measure ring-global invariants, the combined method is model independent. Furthermore, the needed closed-orbit perturbation has only negligible effects on the beam. This has to be compared to the (partly) disruptive influence on the beam if $\beta$ and $\varphi$ is measured by quadrupole variation or determined via ORM analysis [9] (note that for an ORM analysis at DELTA more than 50 orbit perturbations are necessary). In addition, at the DELTA facility methods such as LOCO cannot be used reliably since the combined-function correctors at the quadrupoles produce undesired higher-order multipole fields, so that the given standard lattice model becomes underdetermined. The problem of undesired higher-order multipole fields is also present in many other facilities.

The resolution of the combined method is slightly lower than pure multiturn measurements but better than in the mentioned quasistatic methods. In addition, the resolution of the combined method does not depend on the quality of the given lattice model. However, the combined method may be used to improve a given lattice model. Recently, Huang et al. [4] introduced a method which improves a lattice model with multiturn data through a fitting procedure. The combined method introduced here also provides the needed data at all conventional BPMs as if they were multiturn BPMs, so that an improvement of a given lattice model is also possible with the combined method.

\section{CONCLUSION AND OUTLOOK}

In this work, a proof-of-principle experiment has been described and implemented which shows that modelindependent and fast measurements of linear beam Twiss parameters are possible with much less hardware effort than usually needed for retrieving this information. This was achieved by a combination of analog and multiturn BPMs. The optical functions along the whole ring can then be retrieved using quasistationary closed-orbit perturbation with correctors inside the drift space. This approach is demonstrated in the horizontal oscillation plane but its principles can be extended to the full transverse plane including linear betatron coupling without restrictions (see the Appendix). Although the presented method is model independent, it can be combined with approaches to improve a given lattice model with multiturn data like the approach introduced recently by Huang et al. [4].

In the near future, a fast-orbit feedback system for the DELTA storage ring will be installed (see Ref. [14]) which also includes vertical dipole correctors. Together with the drift space between BPM 39 and BPM 40 (see Fig. 2), it will then be possible to also measure the vertical $\beta$ and $\varphi$ functions at all BPM positions. The correctors and BPMs of this fast-orbit feedback system also enable a very fast application of the closed-orbit perturbation with a kHzrange sampling rate so that the measuring time (here below 1 min) may be reduced by two or 3 orders of magnitude. This fast measurement of the lattice is desirable if, for instance, the lattice parameters are tuned for new purposes like seeded FEL (see Ref. [15]). Furthermore, a bunchby-bunch feedback system will be installed at DELTA which will raise the amount of multiturn data. This will significantly increase the resolution of the method presented here.

\section{ACKNOWLEDGMENTS}

The authors thank P. Hartmann, H. Huck, S. Khan, and $\mathrm{H}$. Rast for valuable discussions.

\section{APPENDIX: GENERALIZATION TO TRANSVERSE MOTION AND BETATRON COUPLING}

For a description of betatron coupling, see also Ref. [16] (for further reading see Ref. [17]). 


\section{Deriving the optical functions of a drift space with multiturn BPMs}

If one considers the four-dimensional transverse phase space, the generalized form of the expected multiturn data for $x$ and $y$ reads

$$
\begin{aligned}
& x_{n j}=\operatorname{Re}\left(X_{j} e^{i \mu_{1} n}+A_{j} e^{i \mu_{2} n}\right), \\
& y_{n j}=\operatorname{Re}\left(B_{j} e^{i \mu_{1} n}+Y_{j} e^{i \mu_{2} n}\right),
\end{aligned}
$$

where the four phasors $X, Y, A, B \in \mathbb{C}$ fully describe the measurable transverse parameters at one BPM $j$, and $\mu_{1,2}=2 \pi Q_{1,2}$ are the measurable betatron phase advances per turn. If a drift space between two BPMs $j=1,2$ is considered, the phasors $X^{\prime}, Y^{\prime}, A^{\prime}, B^{\prime}$ can also be determined from multiturn data by using Eq. (3).

The same definition for $\boldsymbol{F}$ is used as in Ref. [5], leaving out the local BPM index $j$ for convenience, i.e.,

$$
\begin{gathered}
\left(\begin{array}{l}
x_{n} \\
x_{n}^{\prime} \\
y_{n} \\
y_{n}^{\prime}
\end{array}\right)=\boldsymbol{F}\left(\begin{array}{c}
\cos n \mu_{1} \\
\sin n \mu_{1} \\
\cos n \mu_{2} \\
\sin n \mu_{2}
\end{array}\right), \\
\boldsymbol{F}=\left(\begin{array}{cccc}
\operatorname{Re} X & -\operatorname{Im} X & \operatorname{Re} A & -\operatorname{Im} A \\
\operatorname{Re} X^{\prime} & -\operatorname{Im} X^{\prime} & \operatorname{Re} A^{\prime} & -\operatorname{Im} A^{\prime} \\
\operatorname{Re} B & -\operatorname{Im} B & \operatorname{Re} Y & -\operatorname{Im} Y \\
\operatorname{Re} B^{\prime} & -\operatorname{Im} B^{\prime} & \operatorname{Re} Y^{\prime} & -\operatorname{Im} Y^{\prime}
\end{array}\right)=\left(\begin{array}{ll}
\boldsymbol{X} & \boldsymbol{A} \\
\boldsymbol{B} & \boldsymbol{Y}
\end{array}\right) .
\end{gathered}
$$

The four real $2 \times 2$ block matrices $\boldsymbol{X}, \boldsymbol{Y}, \boldsymbol{A}, \boldsymbol{B}$ are also defined. In the uncoupled case, $\boldsymbol{A}$ and $\boldsymbol{B}$ are zero matrices and the determinants of $\boldsymbol{X}$ and $\boldsymbol{Y}$ equal the Courant-Snyder invariants $Y_{x}$ and $Y_{y}$, respectively.

In the coupled case, there are still two Courant-Snyder invariants $Y_{1}, \quad Y_{2}$ of motion which ratio equals $f=|\operatorname{det}(\boldsymbol{X})| /|\operatorname{det}(\boldsymbol{Y})|$. Using this, one can construct a $4 \times 4$ matrix (see Ref. [5])

$$
\boldsymbol{P}=\left(\frac{f}{|\operatorname{det}(\boldsymbol{F})|}\right)^{1 / 4} \boldsymbol{F}\left(\begin{array}{cccc}
f^{-1 / 2} & 0 & 0 & 0 \\
0 & f^{-1 / 2} & 0 & 0 \\
0 & 0 & 1 & 0 \\
0 & 0 & 0 & 1
\end{array}\right)
$$

in which $\boldsymbol{F}$ is normalized in such a way that $\boldsymbol{P}$ does not depend on the invariants but only on the local optical parameters, i.e. $|\operatorname{det}(\boldsymbol{P})|=1$. Unlike in Ref. [5], $\boldsymbol{P}$ is not rotated, so $P_{12}, P_{34}$ not necessarily equal zero and the betatron phase information is included. This does not change the other properties of $\boldsymbol{P}$ that are used in the calculations. Thus, all linear transverse optical parameters, including betatron coupling and phases, are expressed in $\boldsymbol{P}$ in a form similar to action-angle parametrization, e.g., in the uncoupled case $\beta_{x}=P_{11}^{2}+P_{12}^{2}, \beta_{y}=P_{33}^{2}+P_{34}^{2}$ holds.

One can also determine $\boldsymbol{P}$ inside the whole drift in front of $\mathrm{BPM} j=1$. $\boldsymbol{F}$ transforms through the ring and along the longitudinal position $s$ just like a phase space vector $\left(x, x^{\prime}, y, y^{\prime}\right)^{T}$, because the Fourier transformation used to construct $\boldsymbol{F}$ from multiturn data is a linear transformation, i.e.,

$$
\boldsymbol{F}(s)=\boldsymbol{T}\left(s_{1} \rightarrow s\right) \boldsymbol{F}\left(s_{1}\right)
$$

with the $4 \times 4$ section transfer matrix $\boldsymbol{T}$. Since $\boldsymbol{F}$ is known anywhere in the drift section due to its simple transfer matrix, $\boldsymbol{P}$ can be calculated anywhere inside the drift (with the same $f$ ), and thus the local optical parameters at the four corrector positions inside the drift are obtainable.

\section{Closed-orbit perturbation with known values for four correctors}

The $4 \times 4$ one-turn transfer matrix $\boldsymbol{T}_{\text {。 }}$ can be derived from $\boldsymbol{P}$ via (see Ref. [5])

$$
\boldsymbol{T}_{\circ}=\boldsymbol{P}\left(\begin{array}{cccc}
\cos \mu_{1} & \sin \mu_{1} & 0 & 0 \\
-\sin \mu_{1} & \cos \mu_{1} & 0 & 0 \\
0 & 0 & \cos \mu_{2} & \sin \mu_{2} \\
0 & 0 & -\sin \mu_{2} & \cos \mu_{2}
\end{array}\right) \boldsymbol{P}^{-1}
$$

For a closed-orbit perturbation by a horizontal corrector

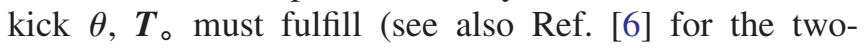
dimensional case)

$$
\boldsymbol{T}_{\mathrm{\circ}} \vec{h}_{\text {corr X }}=\vec{h}_{\text {corr X }}-\theta\left(\begin{array}{l}
0 \\
1 \\
0 \\
0
\end{array}\right)
$$

where $\vec{h}_{\text {corr X }}$ is referring to the four-dimensional phase space vector at the immediate vicinity after the location of the corrector, so that a shift in $x^{\prime}$ direction occurs at the corrector position. Equation (A7) can be solved for $\vec{h}_{\text {corr X }}$ by solving the linear system of equations

$$
\left(\boldsymbol{T}_{\circ}-\mathbf{1}\right) \vec{h}_{\text {corr X }}=-\theta\left(\begin{array}{l}
0 \\
1 \\
0 \\
0
\end{array}\right)
$$

with the $4 \times 4$ identity matrix 1 . Note that different dipole kicks $\theta$ only scale the resulting vector $\vec{h}_{\text {corr } \mathrm{X}}$ but do not change its direction in phase space.

The resulting perturbation vector $\vec{h}$ at any position in the ring can then be expressed as

$$
\vec{h}(s)=\left(\begin{array}{c}
x \\
x^{\prime} \\
y \\
y^{\prime}
\end{array}\right)=\boldsymbol{T}\left(s_{\text {corr X }} \rightarrow s\right) \vec{h}_{\text {corr X }}
$$

for $s>s_{\text {corr X }}$. An analogous calculation can be used for a vertical perturbation $\vec{h}_{\text {corr Y }}$. 
To simplify the following calculations, the orbit perturbations of each of the four correctors are tracked to one reference position $s_{\text {ref }}$ inside the drift using the derived expressions for closed-orbit perturbation. With the four tracked phase space vectors $\vec{h}_{\operatorname{Tr} 1-4}$, each belonging to one corrector, a $4 \times 4$ matrix

$$
\boldsymbol{H}_{\text {corr }}:=\left(\begin{array}{llll}
\vec{h}_{\operatorname{Tr} 1} & \vec{h}_{\operatorname{Tr} 2} & \vec{h}_{\operatorname{Tr} 3} & \vec{h}_{\operatorname{Tr} 4}
\end{array}\right)
$$

can be constructed. This notation is also used for the measurable orbit perturbations $\boldsymbol{H}_{j}$ at BPM $j$. Although it is obvious that only the first and third row of $\boldsymbol{H}_{j}$ can be measured, i.e.

$$
\boldsymbol{H}_{j}=\left(\begin{array}{cccc}
x_{\text {corr X1 }} & x_{\text {corr X2 }} & x_{\text {corr Y1 }} & x_{\text {corr Y2 }} \\
H_{21} & H_{22} & H_{23} & H_{24} \\
y_{\text {corr X1 }} & y_{\text {corr X2 }} & y_{\text {corr Y1 }} & y_{\text {corr Y2 }} \\
H_{41} & H_{42} & H_{43} & H_{44}
\end{array}\right)_{j}
$$

Equation (A9) can then be used to find the relations

$$
\begin{gathered}
\boldsymbol{H}_{j}=\boldsymbol{T}\left(s_{\mathrm{ref}} \rightarrow s_{j}\right) \boldsymbol{H}_{\mathrm{corr}} \\
\boldsymbol{F}_{j}=\boldsymbol{T}\left(s_{\mathrm{ref}} \rightarrow s_{j}\right) \boldsymbol{F}_{\mathrm{ref}}=\boldsymbol{H}_{j} \boldsymbol{H}_{\mathrm{corr}}^{-1} \boldsymbol{F}_{\mathrm{ref}} .
\end{gathered}
$$

The matrix $\boldsymbol{H}_{\text {corr }}$ is only invertible if all of its four column vectors (correctors) are linear independent; this corresponds to the necessary condition of $\Delta \psi \neq n \pi$ in the uncoupled case [see Eq. (A11)]. If one chooses $s_{\text {ref }}=$ $s_{\text {corr X1 }}$, one reduces the computational effort.

Although $\boldsymbol{H}_{j}$ cannot be measured completely at BPM $j$, one can calculate the first and third row of $\boldsymbol{F}_{j}$. Since the invariant fraction $f$ and the four-dimensional invariant $|\operatorname{det}(\boldsymbol{F})|$ are ring-global parameters and can be normalized by using the multiturn BPMs at the ends of the drift space, also the first and third rows of $\boldsymbol{P}_{j}$ at all BPM positions $j$ can be calculated.

This is the same information one would obtain for coupled linear betatron motion if all BPMs $j$ were multiturn capable. Thus, the calculated $\boldsymbol{F}_{j}$ matrices can also be used to obtain multiturn information at all BPMs $j$ by using Eq. (A2) as if all BPMs were multiturn BPMs.

[1] Proceedings of CERN Accelerator School-Course on Beam Diagnostics, edited by D. Brandt (CERN, Geneva, 2009).

[2] M. Minty and F. Zimmermann, Measurement and Control of Charged Particle Beams (Springer, Berlin, Germany, 2003).

[3] H. Wiedemann, Particle Accelerator Physics (Springer, Berlin, Germany, 2007), 3rd ed.

[4] X. Huang, J. Sebek, and D. Martin, Phys. Rev. ST Accel. Beams 13, 114002 (2010).

[5] Y. Luo, Phys. Rev. ST Accel. Beams 7, 124001 (2004).

[6] K. Wille, The Physics of Particle Accelerators: An Introduction (Oxford University Press, Oxford, UK, 2001).

[7] C.-X. Wang, Phys. Rev. ST Accel. Beams 7, 114001 (2004).

[8] M. Negrazus, Ph.D. thesis, Dortmund University, 1994.

[9] J. Safranek, in Proceedings of the Particle Accelerator Conference, Dallas, TX, 1995 (IEEE, Piscataway, NJ, 1995), Vol. 5, pp. 2817-2819.

[10] Bergoz, Multiplexed Beam Position Monitor User's Manual (Bergoz Instrumentation, Saint Genis Pouilly, France).

[11] A. Kosicek, Libera Electron User Manual (Instrumentation Technologies, Solkan, Slovenija, 2008).

[12] The MathWorks Inc., "Matlab R2008a" (2008).

[13] EPICS, "Experimental Physics and Industrial Control System".

[14] P. Hartmann, G. Schuenemann, P. Towalski, D. Schirmer, T. Weis, and S. Khan, in Proceedings of ICALEPCS2009, Kobe, Japan (IEEE, Piscataway, NJ, 2009), pp. 791-793.

[15] S. Khan, in Proceedings of the 23rd Particle Accelerator Conference, Vancouver, Canada, 2009 (IEEE, Piscataway, NJ, 2009), pp. 1144-1146.

[16] D. A. Edwards and L. C. Teng, IEEE Trans. Nucl. Sci. 20, 885 (1973).

[17] R. Calaga, R. Tomás, and A. Franchi, Phys. Rev. ST Accel. Beams 8, 034001 (2005). 